\title{
$g l_{q}(n)$-Covariant Multimode Oscillators and q-Symmetric States
}

\author{
W.-S.Chung \\ Department of Physics and Research Institute of Natural Science, \\ Gyeongsang National University, \\ Jinju, 660-701, Korea
}

\begin{abstract}
In this paper the coherent states and q-symmetric states for $g l_{q}(n)$ covariant multimode oscillator system are investigated.
\end{abstract}

\section{Introduction}

Quantum groups or q-deformed Lie algebra implies some specific deformations of classical Lie algebras.

From a mathematical point of view, it is a non-commutative associative Hopf algebra. The structure and representation theory of quantum groups have been developed extensively by Jimbo [1] and Drinfeld [2].

The q-deformation of Heisenberg algebra was made by Arik and Coon [3], Macfarlane [4] and Biedenharn [5]. Recently there has been some interest in more general deformations involving an arbitrary real functions of weight generators and including q-deformed algebras as a special case [6-10].

Recently Greenberg [11] has studied the following q-deformation of multi mode boson algebra:

$$
a_{i} a_{j}^{\dagger}-q a_{j}^{\dagger} a_{i}=\delta_{i j}
$$


where the deformation parameter $q$ has to be real. The main problem of Greenberg's approach is that we can not derive the relation among $a_{i}$ 's operators at all. Moreover the above algebra is not covariant under $g l_{q}(n)$ algebra. In order to solve this problem we should find the q-deformed multimode oscillator algebra which is covariant under $g l_{q}(n)$ algebra. Recently the Fock space representation of $g l_{q}(n)$-covarinat multimode oscillator system was known by some authers [12]. In this paper we construct the correct form of coherent states for the above mentioned oscillator system and obtain the q-symmetric states generalizing the bosonic states.

\section{Coherent states of $g l_{q}(n)$-covariant oscilla- tor algebra}

$g l_{q}(n)$-covariant oscillator algebra is defined as [12]

$$
\begin{gathered}
a_{i}^{\dagger} a_{j}^{\dagger}=q a_{j}^{\dagger} a_{i}^{\dagger}, \quad(i<j) \\
a_{i} a_{j}=\frac{1}{q} a_{j} a_{i}, \quad(i<j) \\
a_{i} a_{j}^{\dagger}=q a_{j}^{\dagger} a_{i}, \quad(i \neq j) \\
a_{i} a_{i}^{\dagger}=1+q^{2} a_{i}^{\dagger} a_{i}+\left(q^{2}-1\right) \sum_{k=i+1}^{n} a_{k}^{\dagger} a_{k}, \quad(i=1,2, \cdots, n-1) \\
a_{n} a_{n}^{\dagger}=1+q^{2} a_{n}^{\dagger} a_{n}, \\
{\left[N_{i}, a_{j}\right]=-\delta_{i j} a_{j}, \quad\left[N_{i}, a_{j}^{\dagger}\right]=\delta_{i j} a_{j}^{\dagger}, \quad(i, j=1,2, \cdots, n)}
\end{gathered}
$$

where we restrict our concern to the case that $q$ is real and $0<q<1$. Here $N_{i}$ plays a role of number operator and $a_{i}\left(a_{i}^{\dagger}\right)$ plays a role of annihilation(creation) operator. From the above algebra one can obtain the relation 
between the number operators and mode opeartors as follows

$$
a_{i}^{\dagger} a_{i}=q^{2 \sum_{k=i+1}^{n} N_{k}}\left[N_{i}\right]
$$

where $[x]$ is called a q-number and is defined as

$$
[x]=\frac{q^{2 x}-1}{q^{2}-1} .
$$

Let us introduce the Fock space basis $\mid n_{1}, n_{2}, \cdots, n_{n}>$ for the number operators $N_{1}, N_{2}, \cdots, N_{n}$ satisfying

$$
N_{i}\left|n_{1}, n_{2}, \cdots, n_{n}>=n_{i}\right| n_{1}, n_{2}, \cdots, n_{n}>, \quad\left(n_{1}, n_{2}, \cdots, n_{n}=0,1,2 \cdots\right)
$$

Then we have the following representation

$$
\begin{gathered}
a_{i}\left|n_{1}, n_{2}, \cdots, n_{n}>=q^{\sum_{k=i+1}^{n} n_{k}} \sqrt{\left[n_{i}\right]}\right| n_{1}, \cdots, n_{i}-1, \cdots, n_{n}> \\
a_{i}^{\dagger}\left|n_{1}, n_{2}, \cdots, n_{n}>=q^{\sum_{k=i+1}^{n} n_{k}} \sqrt{\left[n_{i}+1\right]}\right| n_{1}, \cdots, n_{i}+1, \cdots, n_{n}>.
\end{gathered}
$$

From the above representation we know that there exists the ground state $\mid 0,0, \cdots, 0>$ satisfying $a_{i} \mid 0,0>=0$ for all $i=1,2, \cdots, n$. Thus the state $\mid n_{1}, n_{2}, \cdots, n_{n}>$ is obtatind by applying the creation operators to the ground state $|0,0, \cdots, 0\rangle$

$$
\left|n_{1}, n_{2}, \cdots, n_{n}>=\frac{\left(a_{n}^{\dagger}\right)^{n_{n}} \cdots\left(a_{1}^{\dagger}\right)^{n_{1}}}{\sqrt{\left[n_{1}\right] ! \cdots\left[n_{n}\right] !}}\right| 0,0, \cdots, 0>.
$$

If we introduce the scale operators as follows

$$
Q_{i}=q^{2 N_{i}}, \quad(i=1,2, \cdots, n)
$$

we have from the algebra (1)

$$
\left[a_{i}, a_{i}^{\dagger}\right]=Q_{i} Q_{i+1} \cdots Q_{n}
$$


Acting the operators $Q_{i}$ 's on the basis $\mid n_{1}, n_{2}, \cdots, n_{n}>$ produces

$$
Q_{i}\left|n_{1}, n_{2}, \cdots, n_{n}>=q^{2 n_{i}}\right| n_{1}, n_{2}, \cdots, n_{n}>
$$

From the relation $a_{i} a_{j}=\frac{1}{q} a_{j} a_{i}, \quad(i<j)$, the coherent states for $g l_{q}(n)$ algebra is defined as

$$
a_{i}\left|z_{1}, \cdots, z_{i}, \cdots, z_{n}>=z_{i}\right| z_{1}, \cdots, z_{i}, q z_{i+1}, \cdots, q z_{n}>
$$

Solving the eq.(9) we obtain

$$
\left|z_{1}, z_{2}, \cdots, z_{n}>=c\left(z_{1}, \cdots, z_{n}\right) \sum_{n_{1}, n_{2}, \cdots, n_{n}=0}^{\infty} \frac{z_{1}^{n_{1}} z_{2}^{n_{2}} \cdots z_{n}^{n_{n}}}{\sqrt{\left[n_{1}\right] !\left[n_{2}\right] ! \cdots\left[n_{n}\right] !}}\right| n_{1}, n_{2}, \cdots, n_{n}>.
$$

Using eq.(5) we can rewrite eq.(10) as

$$
\left|z_{1}, z_{2}, \cdots, z_{n}>=c\left(z_{1}, \cdots, z_{n}\right) \exp _{q}\left(z_{n} a_{n}^{\dagger}\right) \cdots \exp _{q}\left(z_{2} a_{2}^{\dagger}\right) \exp _{q}\left(z_{1} a_{1}^{\dagger}\right)\right| 0,0, \cdots, 0>\text {. }
$$

where q-exponential function is defined as

$$
\exp _{q}(x)=\sum_{n=0}^{\infty} \frac{x^{n}}{[n] !}
$$

The q-exponential function satisfies the following recurrence relation

$$
\exp _{q}\left(q^{2} x\right)=\left[1-\left(1-q^{2}\right) x\right] \exp _{q}(x)
$$

Using the above relation and the fact that $0<q<1$, we obtain the formula

$$
\exp _{q}(x)=\Pi_{n=0}^{\infty} \frac{1}{1-\left(1-q^{2}\right) q^{2 n} x}
$$

Using the normalization of the coherent state, we have

$$
c\left(z_{1}, z_{2}, \cdots, z_{n}\right)=\exp _{q}\left(\left|z_{1}\right|^{2}\right) \exp _{q}\left(\left|z_{2}\right|^{2}\right) \cdots \exp _{q}\left(\left|z_{n}\right|^{2}\right)
$$


The coherent state satisfies the completeness relation

$$
\int \cdots \int\left|z_{1}, z_{2}, \cdots, z_{n}><z_{1}, z_{2}, \cdots, z_{n}\right| \mu\left(z_{1}, z_{2}, \cdots, z_{n}\right) d^{2} z_{1} d^{2} z_{2} \cdots d^{2} z_{n}=I,
$$

where the weighting function $\mu\left(z_{1}, z_{2}, \cdots, z_{n}\right)$ is defined as

$$
\mu\left(z_{1}, z_{2}, \cdots, z_{n}\right)=\frac{1}{\pi^{2}} \Pi_{i=1}^{n} \frac{\exp _{q}\left(\left|z_{i}\right|^{2}\right)}{\exp _{q}\left(q\left|z_{i}\right|^{2}\right)} .
$$

In deriving eq.(15) we used the formula

$$
\int_{0}^{1 /\left(1-q^{2}\right)} x^{n} \exp _{q}\left(q^{2} x\right)^{-1} d_{q^{2}} x=[n] !
$$

\section{3 q-symmetric states}

In this section we study the statistics of many particle state. Let $N$ be the number of particles. Then the N-partcle state can be obtained from the tensor product of single particle state:

$$
\left|i_{1}, \cdots, i_{N}>=\right| i_{1}>\otimes\left|i_{2}>\otimes \cdots \otimes\right| i_{N}>
$$

where $i_{1}, \cdots, i_{N}$ take one value among $\{1,2, \cdots, n\}$ and the sigle particle state is defined by $\left|i_{k}>=a_{i_{k}}^{\dagger}\right| 0>$.

Consider the case that $\mathrm{k}$ appears $n_{k}$ times in the set $\left\{i_{1}, \cdots, i_{N}\right\}$. Then we have

$$
n_{1}+n_{2}+\cdots+n_{n}=\sum_{k=1}^{n} n_{k}=N .
$$

Using these facts we can define the q-symmetric states as follows:

$$
\left|i_{1}, \cdots, i_{N}>_{q}=\sqrt{\frac{\left[n_{1}\right] ! \cdots\left[n_{n}\right] !}{[N] !}} \sum_{\sigma \in \operatorname{Perm}} \operatorname{sgn}_{q}(\sigma)\right| i_{\sigma(1)} \cdots i_{\sigma(N)}>,
$$


where

$$
\begin{aligned}
& \operatorname{sgn}_{q}(\sigma)=q^{R\left(i_{1} \cdots i_{N}\right)} q^{R(\sigma(1) \cdots \sigma(N))}, \\
& R\left(i_{1}, \cdots, i_{N}\right)=\sum_{k=1}^{N} \sum_{l=k+1}^{N} R\left(i_{k}, i_{l}\right)
\end{aligned}
$$

and

$$
R(i, j)= \begin{cases}1 & \text { if } i>j \\ 0 & \text { if } i \leq j\end{cases}
$$

Then the q-symmetric states obeys

$$
\mid \cdots, i_{k}, i_{k+1}, \cdots>_{q}= \begin{cases}q^{-1} \mid \cdots, i_{k+1}, i_{k}, \cdots>_{q} & \text { if } i_{k}<i_{k+1} \\ \mid \cdots, i_{k+1}, i_{k}, \cdots>_{q} & \text { if } i_{k}=i_{k+1} \\ q \mid \cdots, i_{k+1}, i_{k}, \cdots>_{q} & \text { if } i_{k}>i_{k+1}\end{cases}
$$

The above property can be rewritten by introducing the deformed transition operator $P_{k, k+1}$ obeying

$$
P_{k, k+1}\left|\cdots, i_{k}, i_{k+1}, \cdots>_{q}=\right| \cdots, i_{k+1}, i_{k}, \cdots>_{q}
$$

This operator satisfies

$$
P_{k+1, k} P_{k, k+1}=I d, \quad \text { so } \quad P_{k+1, k}=P_{k, k+1}^{-1}
$$

Then the equation (21) can be written as

$$
P_{k, k+1}\left|\cdots, i_{k}, i_{k+1}, \cdots>_{q}=q^{-\epsilon\left(i_{k}, i_{k+1}\right)}\right| \cdots, i_{k+1}, i_{k}, \cdots>_{q}
$$

where $\epsilon(i, j)$ is defined as

$$
\epsilon(i, j)= \begin{cases}1 & \text { if } i>j \\ 0 & \text { if } i=j \\ -1 & \text { if } i<j\end{cases}
$$


The relation (24) goes to the symmetric relation for the ordinary bosons when the deformation parameter $q$ goes to 1 . If we define the fundamental q-symmetric state $\mid q>$ as

$$
|q>=| i_{1}, i_{2}, \cdots, i_{N}>_{q}
$$

with $i_{1} \leq i_{2} \leq \cdots \leq i_{N}$, we have

$$
\| q>\left.\right|^{2}=1 .
$$

In deriving the above relation we used following identity

$$
\sum_{\sigma \in \text { Perm }} q^{2 R(\sigma(1), \cdots, \sigma(N))}=\frac{[N] !}{\left[n_{1}\right] ! \cdots\left[n_{n}\right] !}
$$

The derivation of above formula will be given in Appendix.

\section{Concluding Remark}

In this paper the $g l_{q}(n)$-covariant oscillator algebra and its coherent states are discussed. The q-symmetric states generalizing the symmetric (bosonic) states are obtained by using the $g l_{q}(n)$-covariant oscillators and are shown to be orthonormal. I think that the q-symmetric states will be important when we consider the new statistical field theory generalizing the ordinary one.

\section{Appendix}

In this appendix we prove the relation(25) by using the mathematical induction. Let us assume that the relation (25) holds for $N$. Now we should prove 
that eq.(25) still hold for $N+1$. Let us consider the case that $i$ appears $n_{i}+1$ times. Then we should show

$$
\sum_{\sigma \in \operatorname{Perm}} q^{2 R(\sigma(1), \cdots, \sigma(N+1))}=\frac{[N+1] !}{\left[n_{1}\right] ! \cdots\left[n_{i-1}\right] !\left[n_{i}+1\right] !\left[n_{i+1}\right] ! \cdots\left[n_{n}\right] !}
$$

In this case the above sum can be written by three pieces:

$$
\sum_{j=1}^{i-1} \sum_{\sigma(1)=j}+\sum_{\sigma(1)=i}+\sum_{j=i+1}^{n} \sum_{\sigma(1)=j}
$$

Thus the left hand side of eq.(26) is given by

$$
\begin{aligned}
L H S & =\sum_{j=1}^{i-1} \sum_{\sigma(1)=j} q^{2 R(j, \sigma(2), \cdots, \sigma(n+1))} \\
& +\sum_{\sigma(1)=i} q^{2 R(i, \sigma(2), \cdots, \sigma(n+1))} \\
& +\sum_{j=i+1}^{n} \sum_{\sigma(1)=j} q^{2 R(j, \sigma(2), \cdots, \sigma(n+1))}
\end{aligned}
$$

Then we have

$$
R(j, \sigma(2), \cdots, \sigma(n+1))=\sum_{k=2}^{N+1} R(j, \sigma(k))+R(\sigma(2), \cdots, \sigma(N+1))
$$

where

$$
\sum_{k=2}^{N+1} R(j, \sigma(k))= \begin{cases}n_{1}+\cdots+n_{j-1} & \text { if } j \leq i \\ n_{1}+\cdots+n_{j-1}+1 & \text { if } j>i\end{cases}
$$

Using the above relations the LHS of eq.(26) can be written as

$$
\begin{aligned}
L H S & =\sum_{j=1}^{i-1} q^{2\left(n_{1}+\cdots+n_{j-1}\right)} \frac{[N] !}{\left[n_{i}+1\right] !\left[n_{j}-1\right] ! \Pi_{k \neq i, j}\left[n_{k}\right] !} \\
& +q^{2\left(n_{1}+\cdots+n_{i-1}\right)} \frac{[N] !}{\prod_{k}\left[n_{k}\right] !}
\end{aligned}
$$




$$
+\sum_{j=i+1}^{N+1} q^{2\left(n_{1}+\cdots+n_{j-1}+1\right)} \frac{[N] !}{\left[n_{i}+1\right] !\left[n_{j}-1\right] ! \Pi_{k \neq i, j}\left[n_{k}\right] !}
$$

If we pick up the common factor of three terms of eq.(29), we have

$$
I=J \frac{[N] !}{\left[n_{i}+1\right] ! \Pi_{k \neq i}\left[n_{k}\right] !}
$$

where

$$
\begin{aligned}
J & =\frac{1}{q^{2}-1}\left[\sum_{j=1}^{i-1} q^{2\left(n_{1}+\cdots+n_{j-1}\right)}\left[n_{j}\right]+q^{2\left(n_{1}+\cdots+n_{i-1}\right)}\left[n_{1}+1\right]+\sum_{j=i+1}^{N+1} q^{2\left(n_{1}+\cdots+n_{j-1}+1\right)}\left[n_{j}\right]\right. \\
& =[N+1]
\end{aligned}
$$

Thus we proved the relation (25).

\section{Acknowledgement}

This paper was supported by the KOSEF (961-0201-004-2) and the present studies were supported by Basic Science Research Program, Ministry of Education, 1995 (BSRI-95-2413). 


\section{References}

[1] Jimbo,Lett.Math.Phys.10 (1985) 63;11(1986)247.

[2] V.Drinfeld,Proc.Intern.Congress of Mathematicians (Berkeley, 1986) 78.

[3] M.Arik and D.Coon, J.Math.Phys.17 (1976) 524.

[4] A.Macfarlane,J.Phys.A22(1989) 4581.

[5] L.Biedenharn, J.Phys.A22(1989)L873.

[6] A.Polychronakos, Mod.Phys.Lett.A5 (1990) 2325.

[7] M.Rocek, Phys.Lett.B225 (1991) 554.

[8] C.Daskaloyannis, J.Phys.A24 (1991) L789.

[9] W.S.Chung,K.S.Chung ,S.T.Nam and C.I.Um, Phys.Lett.A183 (1993) 363.

[10] W.S.Chung, J.Math.Phys.35 (1994) 3631.

[11] O.Greenberg, Phys.Rev.D43(1991)4111

[12] R.Jagannathan,et.al., J.Phys.A25(1992) 6429. 